\title{
Assessment of CFD Model Performance for Flows around a Hydraulic Structure of Complex Geometry
}

\author{
Péter Grivalszki ${ }^{1 *}$, Gábor Fleit ${ }^{1}$, Sándor Baranya ${ }^{1}$, János Józsa ${ }^{1,2}$ \\ ${ }^{1}$ Department of Hydraulic and Water Resources Engineering, Faculty of Civil Engineering, Budapest University of Technology and \\ Economics, H-1111 Budapest, Műegyetem rkp. 3., Hungary \\ 2 MTA-BME Water Management Research Group, Hungarian Academy of Sciences, H-1051 Budapest, Széchenyi István tér 9, Hungary \\ * Corresponding author, e-mail: grivalszki.peter@epito.bme.hu
}

Received: 21 June 2020, Accepted: 16 September 2020, Published online: 14 October 2020

\begin{abstract}
Computational Fluid Dynamics (CFD) modeling has become a widely used research tool in the hydraulic engineering community, however, for many problems the numerical model setup and even the selection of the suitable hydrodynamic solver is still a challenging task. This is the case for fine scale analysis of flow features around hydraulic structures of complex geometry, where generation of the 3D grid, application of the correct turbulence modeling approach, or modeling of the free surface all require extensive experience and knowledge. The aim of this paper is to perform a numerical hydrodynamic experiment for a hydraulic structure with complex geometry to assess the model performance, in terms of grid resolution, grid refinement methods as well as turbulence modeling. The open source modeling environment of OpenFOAM is tested and validated against laboratory measurements, moreover, practical recommendations are made for future applications of the numerical solver.
\end{abstract}

Keywords

CFD modeling, OpenFOAM, RANS, complex hydraulic structure, turbulence modeling

\section{Introduction}

The rapid, continuous improvement of computational resources in the last decades implied continuous (still ongoing) developments in computational sciences as well. In fact, such an intensive progress took place in the field of the so called CFD (Computational Fluid Dynamics) modeling. These models are used in mechanical as well as in hydraulic engineering, aiming to solve fluid flow problems of various complexity.

In the field of hydraulic engineering, and river engineering in particular the most frequently used hydrodynamic modeling tools use dimensional simplifications, that is, the flow conditions (velocities) are schematized with cross-sectionally averaged, one-dimensional (1D) or depth-averaged, two-dimensional (2D) velocities using the St Venant and the shallow-water equations, respectively $[1,2]$. The development of $1 \mathrm{D}$ and 2D models looks back to several decades due to the fact that they require less computational capacity and earlier PCs enabled the simulation of simpler problems. However, river flows reportedly can show locally complex, three-dimensional (3D), secondary flow features as well [3], which not only influence the prevailing flow conditions, but also play a fundamental role in sediment transport [3]. In the case of 3D fluid modeling, no cross-sectional or vertical integrating is applied, but the Navier-Stokes equations are solved numerically with various simplifications. Resolving the flow problem along the vertical axis, however, increases computational demands significantly, moreover, requires dedicated numerical methods. Methods of various complexity and flexibility are available for the vertical discretization in 3D models, whose applicability depends on the actual flow problem.

Typical hydraulic engineering problems requiring the resolution of 3D flow phenomena are flows around various hydraulic structures, such as weirs, bridge piers, abutments, etc. Roulund et al. [4], for instance, used Reynolds-averaged Navier-Stokes (RANS) model with $k$ - $\omega$ turbulence closure to investigate flow and scouring around a circular pile, with special focus on the horseshoe and lee wake vortices. They successfully captured all the main features of the scouring process, however, their model did not include direct free surface modeling, which 
is an applicable approach in case of flow Froude number $(F r<0.5)$ conditions. In case of larger Froude numbers, the profile of the free surface has notable influence on the hydrodynamic conditions (and vice versa), hence its proper representation is necessary for accurate scour estimations as well. Baranya et al. [5] also showed the applicability of a rigid lid approach for modeling flow and local scour around bridge piers. A $k-\varepsilon$ turbulence closure was used on a curvilinear nested grid system. Laboratory experiments were used to validate the simulations and quantify velocity and turbulent kinetic energy (TKE) around piers. Baranya et al. [6] successfully extended their analysis to predict scouring around the piers. Kahraman et al. [7] investigated the influence of free-surface variation on the velocity field using numerical simulations of flow around a sharp-nosed pier. They concluded, that the volume of fluid (VOF) models are in better agreement with experimental data than those using the rigid-lid approximation. Their results show that even though the change in free-surface height near the pier is small compared to the approach flow, it still has a significant effect on velocities in front of the pier and in the wake region, also at low Froude numbers. Kara et al. [8] carried out large eddy simulations (LES) to investigate the effects of accurate simulation of the curvilinear water-surface deformation of the flow through a bridge contraction. They also tested the rigid-lid boundary condition for free surface, and compared to level set method (LSM). Bed-shear stresses and first-order statistics were similar for their cases, but the instantaneous turbulence structure and consequently, the second-order statistics, were distinctly different. The correct prediction of the water-surface deformation of such flows is deemed important for the accuracy of their simulations.

Stoesser et al. [9] treated the free surface as a plane of symmetry where zero gradient conditions are applied for the variables parallel to the surface with the wall-normal variables set to zero. Their calculations of primary and secondary flow and boundary shear stresses in a meandering laboratory-scaled channel were successful.

If water level fluctuations and changes are to be accounted for as well, several methods are available. One of the most frequently used is the $\sigma$-coordinate transformation method [10]. Based on this, Baranya et al. [11] made largescale flow analysis at a river confluence, and, validating the results against field measurements, they satisfactorily reproduced the most relevant elements of the confluence hydrodynamics. Haun and Olsen [12] used an algorithm based on the computed pressure field when modeling the flushing process of the Kali Gandaki hydropower reservoir. Thus, the location of the water level was calculated with the Bernoulli equation. An adaptive, non-orthogonal and unstructured grid has been used. The total quantity of flushed out sediments, and the bed deformation were compared with experiments, highlighting a good correspondence between the results.

Ignoring free surface variations or using simplifications like the ones in the studies above, usually enough for fulfill the scope of interests in river, sea or ocean scale, but in case of transient free surface motion, steep surface gradients or complex geometries, multiphase models are to be used. In such models, the governing equations are solved not only for the water phase, but for the air above it as well, allowing for multivalued vertical free surface positions. The most frequently used multiphase modeling method from the mentioned ones is the volume of fluid (VOF) method [13]. Furthermore, methods mentioned above cannot handle flows around geometries, which can separate the water domain vertically. For testing different turbulence models, Salaheldin et al. [14] successfully modelled flow around circular piers, where calculated and measured position of the free surface are in good agreement. Haun et al. [15] modelled flow over trapezoidal broad-crested weir, and compared the effectiveness of VOF with fixed grid, and an algorithm based on the continuity equation and the Marker-and-Cell method, together with an adaptive grid for the water surface. They experienced good correlation between the methods, however, VOF required less time for calculations. For high quality simulation of wave generation and absorption, Higuera et al. [16] used VOF, and with it, they set a new boundary condition apparatus to simulate waves. Jacobsen et al. [17] developed an easy to use application called waves 2 Foam which is a VOF-based numerical wave tank approach.

Another approach for capturing the free surface between multiphase flows is the level set method (LSM) [18]. In case of the LSM, the interface is explicitly defined by using a signed level set function in every computational node, defining the closest distance from the free surface. The LSM based CFD toolbox REEF3D [19] has been successfully used to simulate complex flows around various hydraulic engineering structures $[20,21]$.

In addition to the proper tracking of the free-surface, turbulence modeling also plays a crucial role, when modeling transient complex flows, especially in cases where obstacles of complex geometries are present in the flow. Recently, Bayon-Barrachina and López-Jiménez [22], used 
a RANS model with different two equations turbulence closures to simulate a hydraulic jump properly. Jiang et al. [23] simulated flow over a rectangular broad-crested weir with the same apparatus like Bayon-Barrachina and LópezJiménez [22], but with VOF for free surface capturing. In spite of the encouraging results of the above mentioned studies, a major problem with standard RANS turbulence models is that in two-phase simulations, the effect of the free surface is neglected and high turbulence production occurs due to the high gradient in fluid density. Kamath et al. [21] investigated free surface turbulence damping in several different case studies to validate the applicability of their apparatus. They used RANS equations with $k$ - $\omega$ turbulence closure and LSM for capturing free surface. Devolder et al. [24] published the so called buoyancy modification for $k-\omega$ SST turbulence closure, while Larsen and Fuhrman [25] developed a stabilizing correction method that can be used with $k-\varepsilon$ and $k$ - $\omega$ turbulence closures.

In CFD modeling, the numerical domain is generally split into smaller subdomains of geometric primitives like hexahedra or tetrahedra. The governing equations are discretized and solved inside each of these subdomains. For capturing the effects of a structure with complex geometry, it can be a crucial point of model set-up. With manual mesh setup excellent mesh fitting can be achieved, however, for large domains and highly variable geometry it is time consuming, and exposed to manmade errors. The mesh generation can be classified into two main categories based on the topology of the elements that describe the domain, which is structured (eg.: $[22,23])$ and unstructured (e.g.: $[11,15])$. The advantage of structured mesh is that the points of an elemental cell can be easily addressed and the connectivity is straightforward, but for capturing complex geometries, really fine resolution must be applied. An unstructured mesh can capture complex surfaces, but a cell may have an arbitrary number of neighbouring cells attaching to it, making the data treatment and connection complicated [26]. Hybrid meshes containing structured and unstructured blocks are good compromise to use the benefits of both mesh types.

Considering the above described problems related to CFD modeling of complex flows, it can be stated that the selection of suitable numerical solvers for a given hydraulic engineering task, where 3D flow features, turbulence as well as the profile of free surface all play an important role, is far not straightforward. In this study the VOF-based open-source CFD toolbox OpenFOAM [27] was used to simulate turbulent free surface flow conditions around a hydraulic structure of complex geometry. The aim of the study is to test and quantify the accuracy of different frequently used turbulent models, as well as to test the applicability of various mesh fitting algorithms provided by the snappyHexMesh utility through a series of numerical model simulations. CFD model results are compared with experimental data, allowing for a quantitative error analysis. The herein presented results underline the importance of preliminary model tests when investigating flows around complex structures, as relatively high sensitivity was observed for the choice of the tested numerical methods.

\section{Numerical methods}

In this study, the interIsoFoam solver of the open-source CFD toolbox OpenFOAM was used [27], employing a geometric Volume-of-Fluid (VOF) method for ensuring a sharp interface between two immiscible, incompressible fluids. The governing equations for multiphase solver are the Reynolds-averaged Navier-Stokes equations, expressed by the continuity and momentum equations:

$\nabla \boldsymbol{u}=0$,

$\frac{\partial \boldsymbol{u}}{\partial t}+\boldsymbol{u} \cdot \nabla \boldsymbol{u}=-\frac{1}{\rho} \nabla p+v \nabla^{2} \boldsymbol{u}+\boldsymbol{f}_{\boldsymbol{b}}$

where $\boldsymbol{u}$ is velocity vector, $t$ is the time, $\rho$ is density, $p$ is the pressure, $f_{b}$ is body forces (gravity and surface tension). For capturing the free surface between the two phases, the VOF method has been applied [13]. It is an Eulerian volume tracking method with a step function to differentiate water and air phases. A computational cell with $\alpha=1$ is entirely in the water phase, while $\alpha=0$ means the air phase. The interface is represented with an $\alpha$ value between these values. This $\alpha$ value is used as a weight when calculating fluid properties with only one set of governing equations for the entire flow domain. For example, the density in an arbitrary cell can be written as [28]:

$\rho=\alpha \rho_{\text {liquid }}+(1-\alpha) \rho_{\text {gas }}$.

The VOF method then adds one more equation to be solved for the advection of this volume fraction function $\alpha[28]$ :

$\frac{\partial \alpha}{\partial t}+\nabla \cdot(\alpha \boldsymbol{u})=0$ 
The drawback of the standard VOF method is the smearing of the water surface, therefore it cannot capture a sharp surface separating the phase fractions in a cell, but instead the cell will be filled with uniform mixture of the two phases $(0<\alpha<1)$. OpenFOAM offers two methods for improving the surface sharpness. The Multidimensional Universal Limiter with Explicit Solution (MULES) [29] is a widely used scheme to reduce the smearing [30, 31]. Recently, a geometric VOF method has also been implemented as a part of the isoAdvector [32] solver, which was used in present study. This solver reportedly offers a sharper interface tracking than the MULES $[33,34]$.

The governing RANS equations are closed with twoequation turbulence modeling, through the eddy viscosity concept [35]. The performance of two different turbulence models has been tested, namely the standard $k-\omega$ (where $k$ is turbulence kinetic energy and $\omega$ is the specific rate of dissipation of $k$ ) [36] and the $k$ - $\omega$ Shear Stress Transport (SST) model [37], moreover, the effect of the buoyance modification for the $k-\omega$ SST (proposed by $[24,25]$ ) has also been tested. While the standard $k-\omega$ is still used rather frequently, the $k-\omega$ SST can overcome many of the deficiencies of the standard $k$ - $\omega$ model, offering more accurate solutions. The transport equations solved for $k$ and $\omega$ are:

$$
\frac{\partial(\rho k)}{\partial t}+\nabla \cdot(\rho \boldsymbol{u} k)-\nabla \cdot\left(D_{k} \nabla k\right)=P_{k}-\frac{2}{3} \rho \nabla \cdot \boldsymbol{u} k-\rho \beta^{*} \omega k,(5)
$$

$$
\begin{aligned}
& \frac{\partial(\rho \omega)}{\partial t}+\nabla \cdot(\rho \boldsymbol{u} \omega)-\nabla \cdot\left(D_{\omega} \nabla \omega\right) \\
& =P_{\omega}-\frac{2}{3} \rho \Gamma \nabla \cdot \boldsymbol{u} \omega-\rho \beta \omega^{2}-\rho\left(F_{1}-1\right) C D_{k \omega},
\end{aligned}
$$

where $D_{k}$ and $D_{\omega}$ are effective diffusivities for $k$ and $\omega$, $P_{k}$ and $P_{\omega}$ are production terms in the respective equations, $C D_{k \omega}$ is a limiter function, $\beta^{*}=0.09$ is a constant model parameter, $F_{1}$ is a blending function which is used to achieve smooth transition between two models, $\beta$ and $\Gamma$ are parameters which calculated as a weighted averages of $k-\omega$ and $k-\varepsilon$ parameters (where $\varepsilon$ is the turbulent dissipation rate) using $F_{1}$ as a weight function. The turbulent viscosity is calculated from

$v_{t}=\frac{a_{1} \rho k}{\max \left(a_{1} \omega, b_{1} F_{23} \sqrt{2} S\right)}$,

where $a_{1}=0.31$ and $b_{1}=1.0$ are constant parameters, $F_{23}$ is a bounding function and $S$ is the magnitude of the rateof-strain tensor.
OpenFOAM uses the finite volume method (FVM) for discretization [38]. The FVM is implemented by integrating all the terms in the equations over a specified control volume and relating the volume integrals to the surface integrals using Gauss's theorem. The surface and volume integrals are both evaluated with mid-point integration approximation resulting in second-order accuracy. The Laplacian and divergence terms are handled with the Gaussian linear corrected and Gaussian linear schemes. The implicit, first order and transient Euler scheme is used for the time marching.

The snappyHexMesh utility provides three refinement levels (Fig. 1) for mesh morphology at the solid boundary [27]. On the first level, the so-called castellated mesh starts with a hexahedral grid, cutting out the geometry of interest from it with optional refinement, leaving orthogonal edges at the solid boundary. Secondly, snapping makes morphing iterations on the surface with breaking the orthogonality to get a smooth boundary. In the third step, additional layers parallel to the boundary can be added which is often used in mechanical engineering where the turbulent shear layer is to be resolved. In this study an analysis is performed to see if the different fitting methods on a complex geometry with the same spatial resolution affect the hydrodynamic solution.

\section{Case study}

\subsection{The CALTROPe-project}

According to the most recent scientific results, the main reasons of the ongoing, global climate change are the excessive greenhouse gas emissions [39]. Among many adverse effects, sea level rising (along with the biased sediment balance of rivers unsustainable management) entails territorial losses in many river estuaries (e.g. Mekong [40], Mississippi [41], etc.). The CALTROPe project aims to resolve this problem through an easy-applicable structure-system, which works in an organic, nature-oriented way against territorial losses. The structure itself (Fig. 2) is made partly by concrete, and partly by local natural

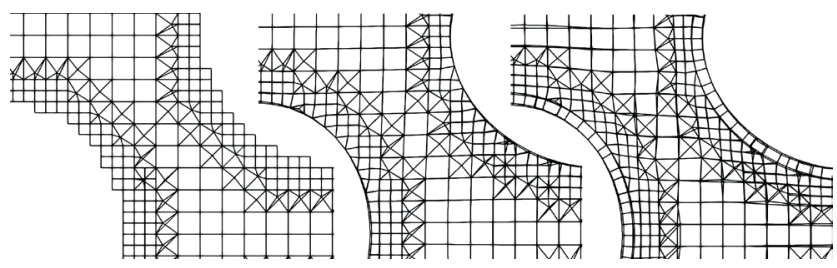

Fig. 1 The three refinement level of snappyHexMesh utility. From left to right: castellated mesh, snapped mesh, add-layers mesh 
side view
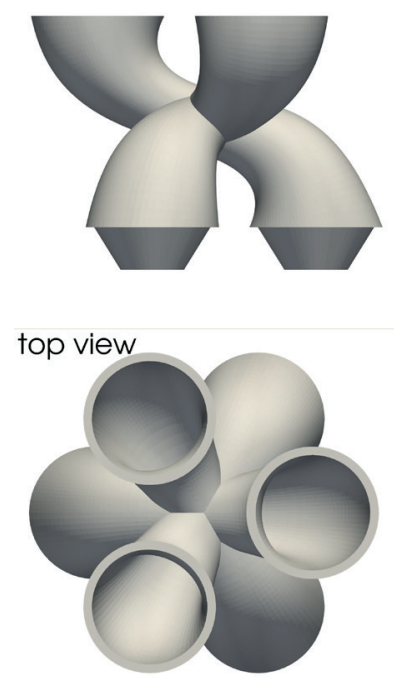

front view

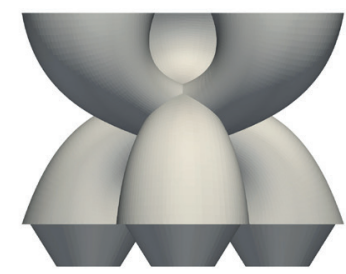

$3 \mathrm{D}$ view

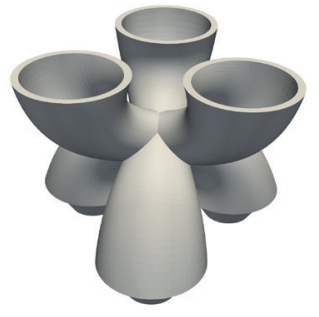

Fig. 2 The CALTROPe

materials. It has three legs and planned to build $1 \mathrm{~m}$ high. Due to its modularity, a shore protection system built from the CALTROPe structures could be installed in river estuaries in some various arrangements. River would flow partly near these arrangements, partly between the structure legs, where the flow velocity is expected to increase, causing scouring and deposition issues.

These hollow structures of limited design age (few years) would serve as habitats for mangrove samplings until they grow and takes root, then would decompose, leaving a fully natural shore protection system behind [42, 43]. The semi-natural system adapts the changes in nature and could help natural sedimentation in coastal areas.

These structures are to be exposed to various kind of hydrodynamic effects, such as the spatially and temporally varying flow field of the incoming river, tidal effects or the dynamic wind induced waves of the sea. It is therefore of crucial importance, for the design of these structures, to possess an investigation tool that is capable to reveal the local scale hydrodynamic impacts. In this study, the flow around a scaled prototype of an individual CALTROPe structure was analyzed both with laboratory experiments and computational tools, but with a main focus on the latter.

\subsection{Laboratory experiments}

Laboratory experiments were performed at the research flume of the Department of Hydraulic and Water Resources Engineering, Budapest University of Technology and Economics. A single 1:10 scaled CALTROPe structure (0.1 m height) made by medical plaster was placed in the midline of the $0.50 \mathrm{~m}$ wide flume in an axially symmetrical way. A constant discharge of $Q=0.008 \mathrm{~m}^{3} \mathrm{~s}^{-1}$ was ensured with a pump system, while the tailwater level was kept at $h=0.08 \mathrm{~m}$ with a tilting weir at the end of the flume. These flow conditions were set to provide slightly turbulent flow conditions with Reynolds number range around $\mathrm{Re} \approx 12000$. It is important to note that the main goal of the paper was to demonstrate the CFD model capabilities through a thorough model test and no upscaling of the modelled flow field to prototype scale is proposed. Consequently, the detailed analysis of the near wall regions was unnecessary, and the main focus of the flow field analysis was on the turbulent region instead.

Single-point velocity data was recorded using an Acoustic Doppler Velocimeter (ADV, Nortek Vectrino) in a high number of points around the structure. Due to its geometrical properties, the sampling volume of the probe is in a distance of $5 \mathrm{~cm}$ from the receiver emitter. Considering this limitation and the $0.08 \mathrm{~m}$ water depth, three nearbed points $(z=0.005,0.015,0.025 \mathrm{~m})$ of 14 verticals were sampled (Fig. 3).

The sampling rate of the ADV was set to $16 \mathrm{~Hz}$, which ensured the logging of turbulent velocity fluctuations as well. Erroneous velocity data (spikes) were removed using a method based on a bivariate kernel density function, [44, 45], and the removed data points were replaced via linear interpolation. In addition to the evaluation of time-averaged velocity vectors, the high-frequency measurements were also used to calculate pointwise values of turbulent kinetic energy (TKE) as:

$T K E=\frac{1}{2}\left(\overline{u_{x}^{\prime 2}}+\overline{u_{y}^{\prime 2}}+\overline{u_{z}^{\prime 2}}\right)$,

where $u_{x}^{\prime}, u_{y}^{\prime}, u_{z}^{\prime}$ are the fluctuating components of the 3D velocity vectors derived via Reynolds-decomposition.

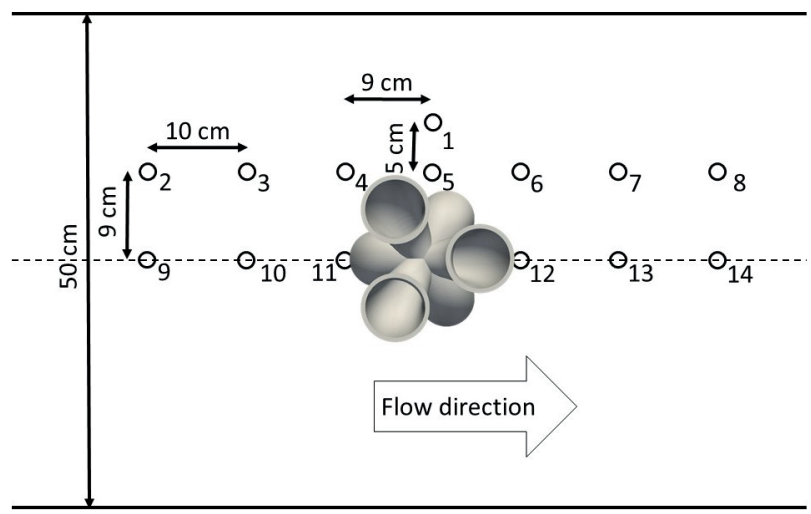

Fig. 3 Layout for laboratory experiments 


\subsection{Numerical setups}

Numerical simulations were performed with the opensource CFD tool OpenFOAM. The model setup was identical to the experimental one, including scale and boundary conditions as well. The model domain is $2 \mathrm{~m}$ long, $0.5 \mathrm{~m}$ wide and $0.2 \mathrm{~m}$ high $-0.08 \mathrm{~m}$ for water phase and $0.12 \mathrm{~m}$ for air phase. Constant inflow discharge $\left(Q=0.008 \mathrm{~m}^{3} \mathrm{~s}^{-1}\right)$ was ensured using the outletPhaseMeanVelocity boundary condition, which sets water level through holding the mean water-phase velocity of $0.2 \mathrm{~ms}^{-1}$. In order to evaluate the performance of i) four mesh resolutions (i.e. grid convergence study) ii) three mesh fitting methods; and iii) three turbulence closures a reference model setup was defined (Table 1). Fig. 4 shows instantaneous velocity streamlines around the CALTROPe.

A recirculation zone and vortex shedding is observed on the downstream side of the obstacle, as well as a periodically moving jet between the structure legs.

Mesh convergence analysis was performed to verify the applied spatial resolution in the reference model, with hexahedron cells of $\Delta x=10.0,7.5,5.0$, and $4.0 \mathrm{~mm}$, which are refined to their half at CALTROPe boundary (model variatons G1, G2, REF, G3 from the coarsest to the finest).

For mesh fitting analysis, all three features of snappyHexMesh, introduced above, were tested. At the add-layer mesh version only one additional layer was implemented due to computational limitations. (model variations M1 (castellated mesh) REF (snapped mesh) and M2 (add layer mesh)).

The performance of three frequently used $k$ - $\omega$-based turbulence closures were tested for the reproduction of main flow features as well as turbulence characteristics. The standard $k-\omega$, the $k-\omega \mathrm{SST}$, and the $k-\omega \mathrm{SST}$ with

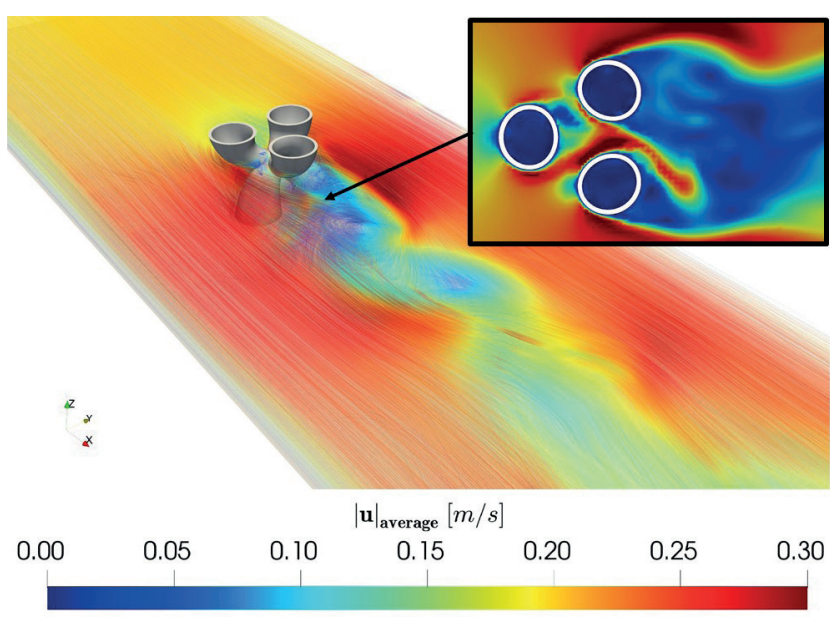

Fig. 4 Instantaneous velocity distribution represented with streamlines (reference model setup) a special multiphase stabilizing correction method by Devolder et al. [24] and Larsen and Fuhrman [25] were tested (buoyancy modification). The latter aims to resolve the problem of turbulence overproduction near the free surface by including a buoyancy term in the transport equations (model variation T1 for $k-\omega$, T2 for $k-\omega$ SST and REF for $k-\omega \mathrm{SST}$ with buoyancy modification).

In the reference model, the resolution of the hexahedral mesh $(\Delta x=5.0 \mathrm{~mm})$, which is further refined to $2.5 \mathrm{~mm}$ in the direct proximity of the CALTROPe surface. The mesh is snapped on the CALTROPe boundary, which means breaking the orthogonality to get a smooth boundary surface. The buoyancy modified $k-\omega$ SST turbulence model have been applied for turbulence closure. The root mean square error (RMSE) was used to quantify the performance of the model variants, through the comparison of measured and modeled horizontal velocity components $\left(u_{x}, u_{y}\right)$ and TKE. Large scale turbulence features (vortex shedding, Fig. 3) are resolved in the transient CFD results, hence the turbulence closure only accounts for the smaller scale fluctuations. Considering that the ADV data inherently contain both scales, the numerical TKE values $\left(T K E_{\text {mod }}\right)$ were derived from the combination of transient velocity fluctuations and modeled turbulent kinetic energy $(k)$ values:

$$
T K E_{\text {mod }}=k+\frac{1}{2}\left(\overline{u_{x}^{2}}+\overline{u_{y}^{\prime 2}}+\overline{u_{z}^{\prime 2}}\right) .
$$

\section{Results}

\subsection{Grid convergence}

Results of the grid convergence study is presented in Fig. 5. (For the location of the measurement verticals, please see Fig. 3.) It is observed, that the steady, logarithmic flow velocity profile at the upstream side of the obstacle (vertical 3) is rather well reproduced at all spatial resolutions. Despite the symmetric geometry, the automatic mesh generation algorithm in the snappyHexMesh tool occasionally provided asymmetric computational grids, which was found to result in erroneous, also asymmetric hydrodynamic solutions: the jet between the structure legs attaches to one of the legs, losing its oscillatory nature. This feature corrupted the time-averaged velocity distributions as well. In order to resolve this problem, an additional row of cells was added width-wise, which replaced the cell faces in the longitudinal symmetry plane of the numerical channel with a cell center.

Vertical 8 is located in the shear layer between high velocity and the recirculation zone, whose numerical representation is believed to be rather challenging. 

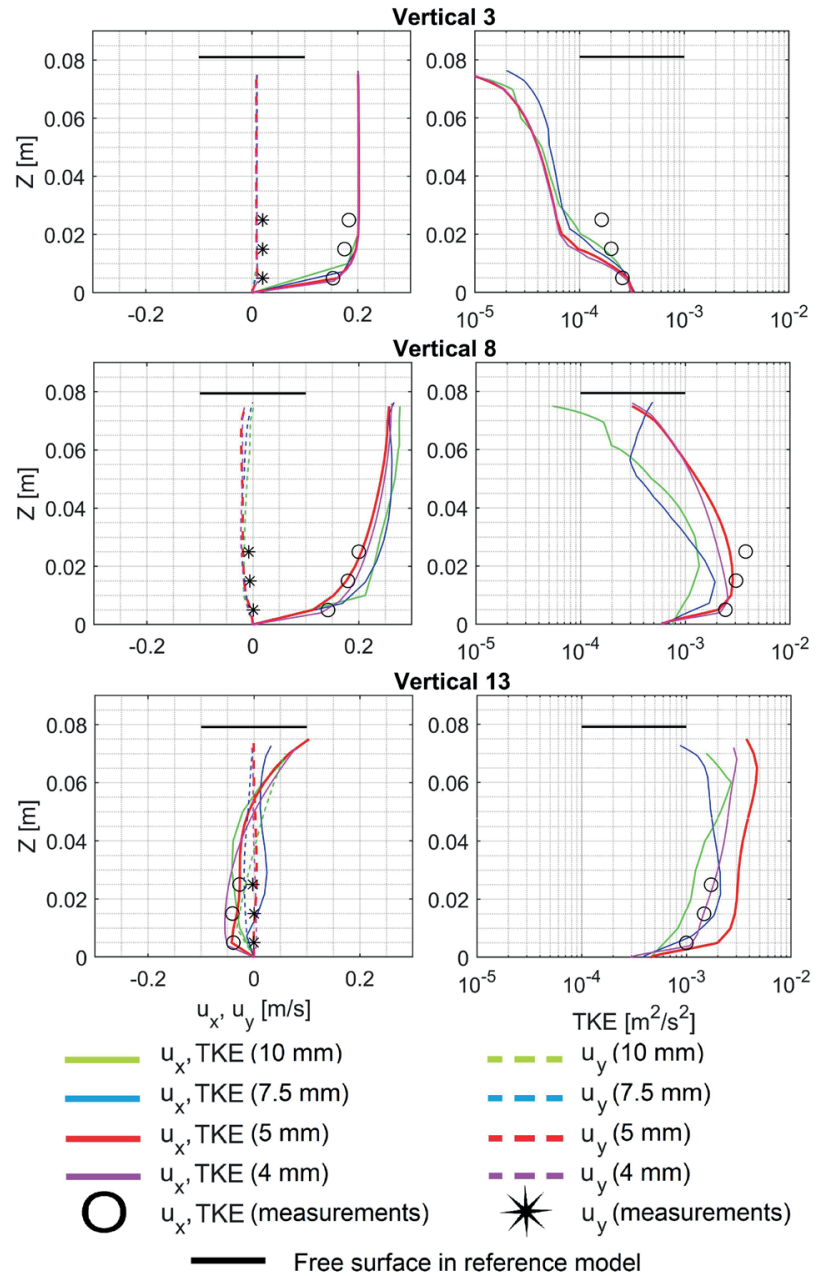

Fig. 5 Vertical velocity and TKE profiles from probes of different flow regimes for mesh resolution analysis

The observed agreement in case of the two finest model variants (REF, G3) is considered very reasonable, while the lack of sufficient cells in the vertical direction results in notable inaccuracies on the coarser meshes. Similarly, in case of the recirculation zone (vertical 13) the two finer model variants provide the best approximation for the flow velocities, however, a slightly contradictory behavior is observed in case of the TKE, where notable overprediction occurs.

In terms of overall accuracy, the gain with the finest resolution (compared to the reference setup) is not that notable with respect to the massive increase of computational demand. On a personal computer (PC) with 16 logical processors with $3.60 \mathrm{GHz}$ basic speed and $32 \mathrm{~Gb}$ random access memory (RAM) the coarsest simulation took only a couple of hours, while the finest is about 2 weeks. Fig. 6 shows the RMSE of the different cases for the measured points. Results show the expected gradual improvements achieved with mesh refinement, especially in case of $u_{x}$.

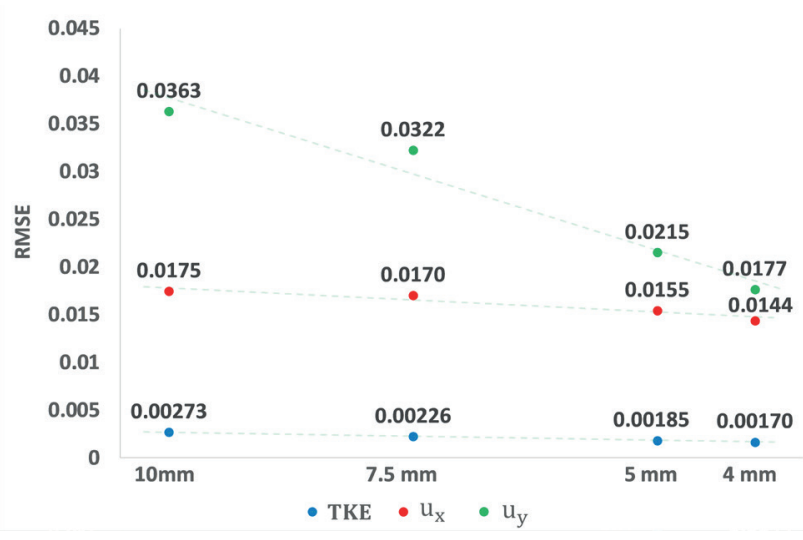

Fig. 6 RMSE values for mesh resolution sensitivity analysis

The authors acknowledge, that additional mesh refinement might further increase model accuracy, however, the general hydraulic behavior of the structure is well represented with the reference model already. In order to optimize resolution and computational time, more advanced dynamic, flow adaptive meshing methods should also be considered [27].

\subsection{Sensitivity to mesh fitting}

Considering the fact, that mesh fitting is only applied at the walls of the obstacle, its effect on the upstream section of the channel was expected to be marginal, which is well-observable in Fig. 7 (vertical 3). In the shear layer, the castellated and snapped meshes show almost identical behavior for both velocities and TKE, however, the additional parallel cell layer around the structure, which is believed to be the most sophisticated method, provides notable worse results. Similar behavior is observed in the recirculation zone (vertical 13), where the strength of the recirculation current is underestimated in model variant M2. With respect to TKE, the most primitive meshing method (M1) provides the best agreement with the experimental data. The contradictory behavior of the add-layers mesh is noted. In mechanical engineering such meshing is often used to resolve the turbulent boundary layer around solid surfaces, however, in such cases multiple and very fine resolution layers are employed. Considering the aims of this study, and CFD modeling in hydraulic engineering in general, such a refinement is usually unnecessary. The presented results suggest, that in cases, where the computational resources do not allow such multilayer refinement at the solid boundary, the applicability of add-layers mesh with a single parallel cell layer is not recommended, despite the fact that it is believed to be the most advanced meshing method from the tested ones. 
RMSE shows that castellated mesh produces the best results in general, however, difference compared to snapped mesh is relatively small (Fig. 8).

\subsection{Effect of turbulence modeling}

In terms of turbulence modeling, the underperformance of the standard $k$ - $\omega$ model is the most obvious (Figs. 9 and 10).
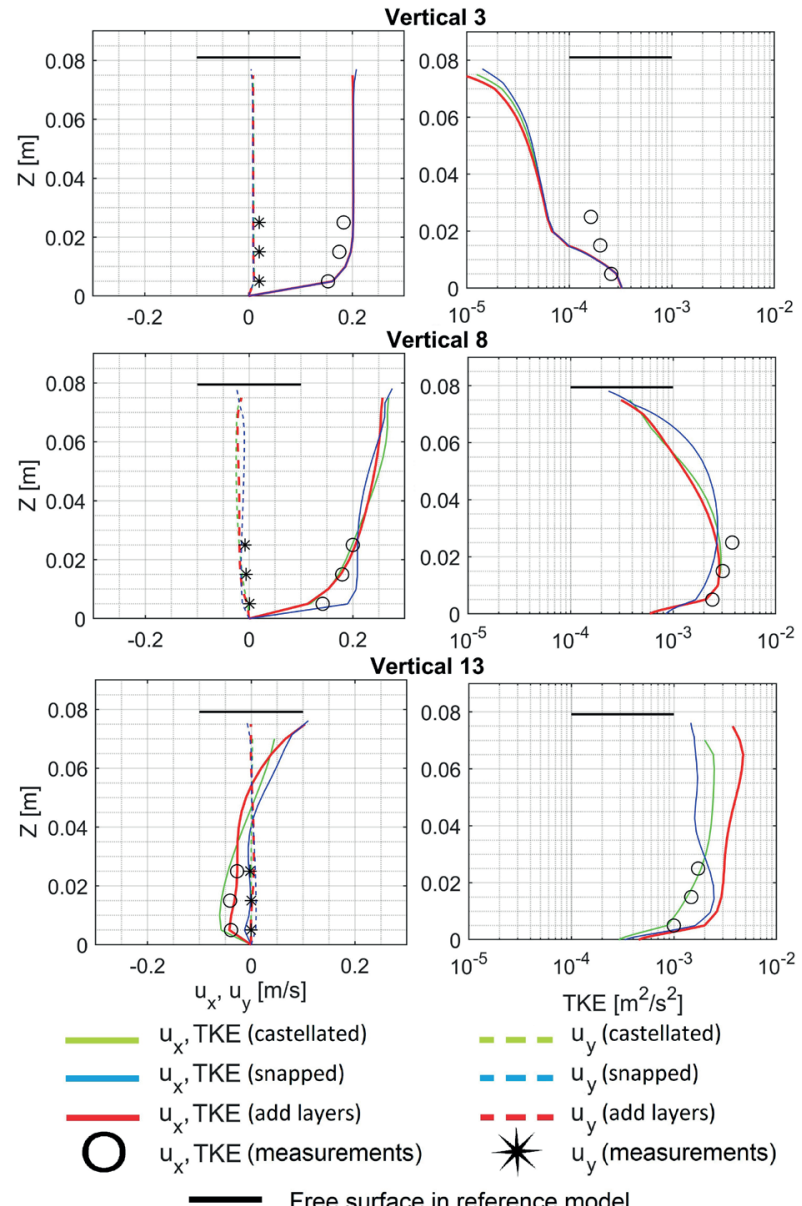

Fig. 7 Vertical velocity and TKE profiles from probes of different flow regimes for mesh fitting analysis

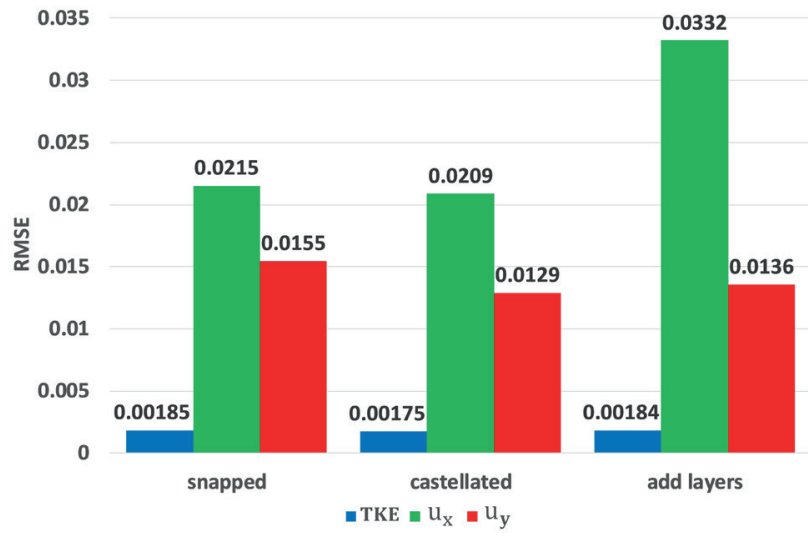

Fig. 8 RMSE values for mesh fitting sensitivity analysis
The approaching, logarithmic flow profile is rather accurately reproduced with all turbulence closures, however, the $k$ - $\omega$ model shows a very notable overproduction of TKE in this area. Regarding the near-bed (measured) section of vertical 3, the $k-\omega$ SST and the modified $k-\omega$ SST models show rather similar results, the physically unrealistic overproduction of turbulence in the proximity of
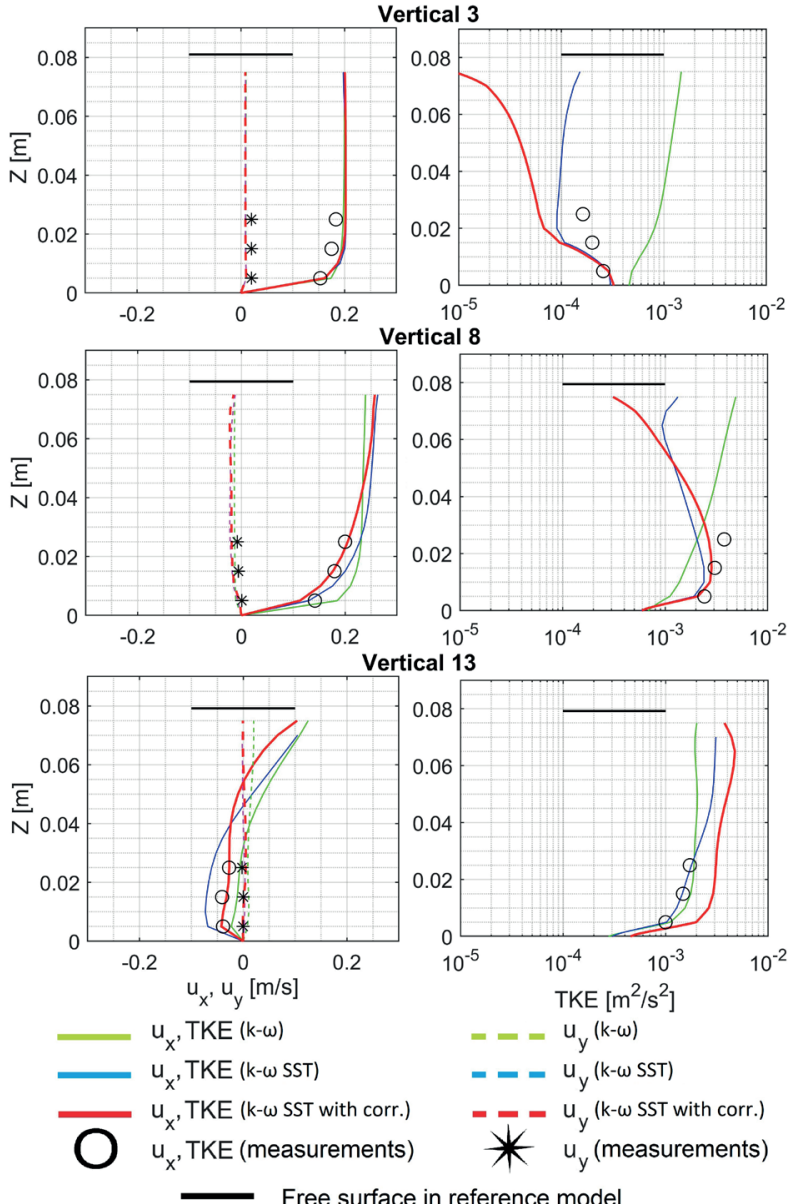

Fig. 9 Vertical velocity and TKE profiles from probes of different flow regimes for different turbulence models

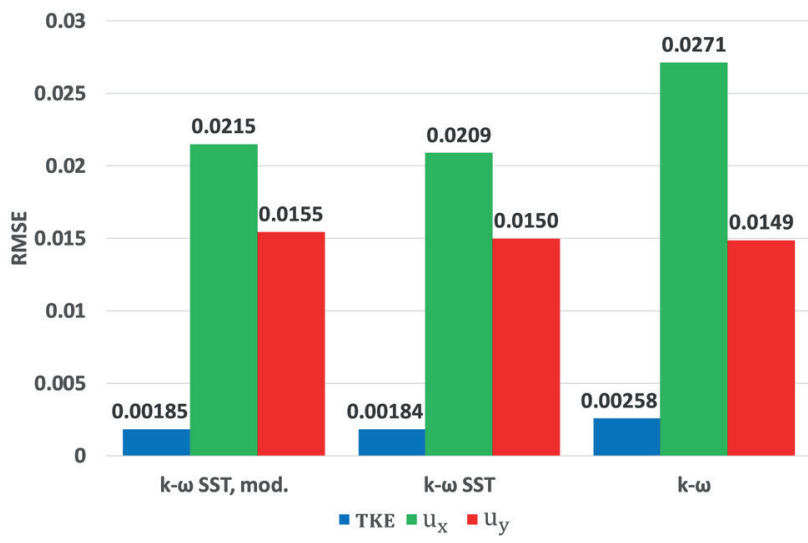

Fig. 10 RMSE values for different turbulence models 
the free surface is observed in case of the former, while the buoyance modification in the latter seems to resolve this issue. While measurements are not available to the near surface region of the flow, considering the expected velocity profile in such a unidirectional, log-profile flow, the authors believe that the buoyancy modified turbulence model provides the more plausible results in this vertical.

Nevertheless, in the recirculation zone (vertical 13) a rather contradictory behavior is observed. While the reference model (using the modified $k$ - $\omega$ SST model) provides the best approximation for the horizontal velocities, the near-bed TKE is notably overpredicted compared to the experimental results and the two inferior turbulence models.

Despite of the differences, calculating RMSE for all verticals reveals that the negative effect of the modification is negligible globally, hence its use is recommended, especially if we have a scope of interest in the near free-surface region (Fig. 10). The most relevant parameters of the compared numerical model setups, as well as the RMSE values are presented in Table 1.

\section{Summary and conclusions}

In this study, flow of constant discharge around a hydraulic structure of complex geometry was investigated with the open-source CFD tool OpenFOAM. In order to verify the numerical results and to ensure the quantitative comparability of various numerical setups, laboratory experiments were performed with an ADV. The high-frequency velocity data was used to calculate pointwise, time-averaged velocity vectors as well as to derive TKE.

A set of numerical models were built with identical geometry and boundary conditions to the experimental setup. A grid convergence study was performed to find the necessary spatial mesh resolution for the adequate representation of the prevailing flow conditions.
Three automatized mesh-fitting algorithms of the snappyHexMesh utility were used to generate computational grids of different complexity and their effect on model accuracy was quantified. The most advanced (add layers) method showed the worst performance, which is probably a reason of the relatively coarse resolution. In mechanical engineering practice, where the near-wall boundary zone has a more emphasized role, such meshing is often used, however, with further refinements and multiple layers. In case of hydraulic engineering, such refinements are often not remunerative, and thus, the application of such meshing options on coarser resolutions are not necessary advised.

The performance of three widely used variants of the $k$ - $\omega$ turbulence model (standard $k-\omega ; k-\omega$ SST; buoyancymodified $k$ - $\omega$ SST) was tested for the prediction of highly turbulent flow conditions around an obstacle of complex geometry.

Results emphasized the relevance of advanced turbulence modeling solutions (sing the $k$ - $\omega$ SST model). The buoyancy modified version of the $k-\omega$ SST model aims to resolve the problem with turbulence overproduction at the proximity of the free surface in case of wave simulations, however, the results presented in this paper also showed its relevance for hydraulic engineering problems as well. It is noted, that the experimental setup did not allow for the sampling of near-surface points of the verticals, hence the beneficial effect of the buoyancy modified $k-\omega$ SST model could not be quantified.

One of the main goals of CFD modeling in hydraulic engineering is to replace costly and time-consuming physical model experiments. However, the results of this study underlined the relevance of such experimental work, more specifically, the advantages of combined hybrid model systems. The relatively high sensitivity of CFD models to the

Table 1 All model setups and root mean square errors compared to measurements

\begin{tabular}{lccccc}
\hline$\#$ & Mesh resolution & Mesh fitting method & turbulence modeling & RMSE Ux & RMSE Uy \\
\hline G1 & $10 \mathrm{~mm}$ & snapped mesh & modified $k$ - $\omega$ SST & 0.0363 & 0.0175 \\
G2 & $7.5 \mathrm{~mm}$ & snapped mesh & modified $k$ - $\omega$ SST & 0.0322 & 0.00273 \\
REF & $5 \mathrm{~mm}$ & snapped mesh & modified $k$ - $\omega$ SST & 0.0215 & 0.00226 \\
G3 & $4 \mathrm{~mm}$ & snapped mesh & modified $k$ - $\omega$ SST & 0.0177 & 0.00185 \\
M1 & $5 \mathrm{~mm}$ & castellated mesh & modified $k$ - $\omega$ SST & 0.0209 & 0.0144 \\
M2 & $5 \mathrm{~mm}$ & add layers mesh & modified $k$ - $\omega$ SST & 0.0332 & 0.0129 \\
T1 & $5 \mathrm{~mm}$ & snapped mesh & $k$ - $\omega$ SST & 0.00170 & 0.0136 \\
T2 & $5 \mathrm{~mm}$ & snapped mesh & $k$ - $\omega$ & 0.00184 & 0.0150 \\
\hline
\end{tabular}


choice of meshing and other numerical methods/parameters, the relevance of preliminary model test is emphasized, however, once a case-specifically well-verified, robust computational model is at hand, the role of numerical modeling is strengthened, as they offer faster and much cheaper analysis compared to physical models, especially if a large number of model variants are to be assessed. It is also noted that CFD modeling not only offers the spatially more extensive analysis of the hydrodynamic characteristics, but also allows for the evaluation of flow variables in areas where conventional (ADV, Particle Image Velocimetry, Laser Doppler Velocimetry, etc.) measurements cannot be performed due to spatial or other restrictions.

\section{References}

[1] Chow, V. T. "Open channel hydraulics", McGraw-Hill, New York, NY, USA, 1959.

[2] Akan, A. O. "7 Bridge hydraulics", In: Open channel hydraulics, Elsevier, Oxford, UK, 2006, pp. 266-314. https://doi.org/10.1016/B978-075066857-6/50008-4

[3] Blanckaert, K., Graf, W. H. "Mean Flow and Turbulence in OpenChannel Bend", Journal of Hydraulic Engineering, 127(10), pp. 835-847, 2001.

https://doi.org/10.1061/(ASCE)0733-9429(2001)127:10(835)

[4] Roulund, A., Mutlu Sumer, B., Fredsøe, J., Michelsen, J. "Numerical and experimental investigation of flow and scour around a circular pile", Journal of Fluid Mechanics, 534, pp. 351-401, 2005 https://doi.org/10.1017/S0022112005004507

[5] Baranya, S., Olsen, N. R. B., Stoesser, T., Sturm, T. W. "ThreeDimensional Rans Modeling of Flow Around Circular Piers using Nested Grids", Engineering Applications of Computational Fluid Mechanics, 6(4), pp. 648-662, 2012. https://doi.org/10.1080/19942060.2012.11015449

[6] Baranya, S., Olsen, N. R. B., Stoesser, T., Sturm, T. W. "A nested grid based computational fluid dynamics model to predict bridge pier scour", Proceedings of the Institution of Civil Engineers - Water Management, 167(5), pp. 259-268, 2014.

https://doi.org/10.1680/wama.12.00104

[7] Kahraman, R., Riella, M., Tabor, G. R., Ebrahimi, M., Djordjević, S., Kripakaran, P. "Prediction of flow around a sharp-nosed bridge pier: influence of the Froude number and free-surface variation on the flow field", Journal of Hydraulic Research, 58(4), pp. 582-593, 2020 .

https://doi.org/10.1080/00221686.2019.1631223

[8] Kara, S., Kara, M. C., Stoesser, T., Sturm, T. W. "Free-Surface versus Rigid-Lid LES Computations for Bridge-Abutment Flow", Journal of Hydraulic Engineering, 141(9), Article number: 04015019, 2015. https://doi.org/10.1061/(ASCE)HY.1943-7900.0001028

[9] Stoesser, T., Ruether, N., Olsen, N. R. B. "Calculation of primary and secondary flow and boundary shear stresses in a meandering channel", Advances in Water Resources, 33(2), pp. 158-170, 2010. https://doi.org/10.1016/j.advwatres.2009.11.001

\section{Acknowledgements}

The first and the second authors acknowledge the support of the UNKR-19-3, while the third author the support of the ÚNKP-19-4 New National Excellence Program of the Ministry for Innovation and Technology. The third author acknowledges the support of the Bolyai János research fellowship of the Hungarian Academy of Sciences. Support of grant BME FIKP-VÍZ by EMMI is also kindly acknowledged. The authors acknowledge the funding of the OTKA FK 128429 grant. Special thanks to VITUKI Hungary Kft. and S39' Hybrid Design Manufacture Kft.

[10] Phillips, N. A. "A coordinate system having some special advantages for numerical forecasting", Journal of Meteorology, 14(2), pp. 184-185, 1957. https://doi.org/10.1175/1520-0469(1957)014<0184:ACSHSS>2.0.CO;2

[11] Baranya, S., Olsen, N. R. B., Józsa, J. "Flow Analysis of a River Confluence with Field Measurements and Rans Model with Nested Grid Approach", River Research and Applications, 31(1), pp. 28-41, 2013.

https://doi.org/10.1002/rra.2718

[12] Haun, S., Olsen, N. R. B. "Three-dimensional numerical modelling of the flushing process of the Kali Gandaki hydropower reservoir", Lakes \& Reservoirs: Research and Management, 17(1), pp. 25-33, 2012.

https://doi.org/10.1111/j.1440-1770.2012.00491.x

[13] Hirt, C. W., Nichols, B. D. "Volume of fluid (VOF) method for the dynamics of free boundaries", Journal of Computational Physics, 39(1), pp. 201-225, 1981. https://doi.org/10.1016/0021-9991(81)90145-5

[14] Salaheldin, T. M., Imran, J., Chaudhry, M. H. "Numerical Modeling of Three-Dimensional Flow Field Around Circular Piers", Journal of Hydraulic Engineering, 130(2), pp. 91-100, 2004. https://doi.org/10.1061/(ASCE)0733-9429(2004)130:2(91)

[15] Haun, S., Olsen, N. R. B., Feurich, R. "Numerical Modeling of Flow Over Trapezoidal Broad-Crested Weir", Engineering Applications of Computational Fluid Mechanics, 5(3), pp. 397-405, 2011. https://doi.org/10.1080/19942060.2011.11015381

[16] Higuera, P., Lara, J. L., Losada, I. J. "Realistic wave generation and active wave absorption for Navier-Stokes models: Application to OpenFOAM®", Coastal Engineering, 71, pp. 102-118, 2013. https://doi.org/10.1016/j.coastaleng.2012.07.002

[17] Jacobsen, N. G., Fuhrman, D. R., Fredsøe, J. "A wave generation toolbox for the open-source CFD library: OpenFoam ${ }^{\circledR}$ ", International Journal for Numerical Methods in Fluids, 70(9), pp. 1073-1088, 2012. https://doi.org/10.1002/fld.2726

[18] Osher, S., Sethian, J. A. "Fronts propagating with curvature-dependent speed: Algorithms based on Hamilton-Jacobi formulations", Journal of Computational Physics, 79(1), pp. 12-49, 1988. https://doi.org/10.1016/0021-9991(88)90002-2 
[19] Bihs, H., Kamath, A., Alagan Chella, M., Aggarwal, A., Arntsen, Ø. A. "A new level set numerical wave tank with improved density interpolation for complex wave hydrodynamics", Computers \& Fluids, 140, pp. 191-208, 2016. https://doi.org/10.1016/j.compfluid.2016.09.012

[20] Fleit, G., Baranya, S., Bihs, H. "CFD Modeling of Varied Flow Conditions Over an Ogee-Weir", Periodica Polytechnica Civil Engineering, 62(1), pp. 26-32, 2018. https://doi.org/10.3311/PPci.10821

[21] Kamath, A., Fleit, G., Bihs, H. "Investigation of Free Surface Turbulence Damping in RANS Simulations for Complex Free Surface Flows", Water, 11(3), Article number: 456, 2019. https://doi.org/10.3390/w11030456

[22] Bayon-Barrachina, A., López-Jiménez, P. A. "Numerical analysis of hydraulic jumps using OpenFOAM", Journal of Hydroinformatics, 17(4), pp. 662-678, 2015. https://doi.org/10.2166/hydro.2015.041

[23] Jiang, L., Diao, M., Sun, H., Ren, Y. "Numerical Modeling of Flow Over a Rectangular Broad-Crested Weir with a Sloped Upstream Face", Water, 10(11), Article number: 1663, 2018. https://doi.org/10.3390/w10111663

[24] Devolder, B., Rauwoens, P., Troch, P. "Application of a buoyancy-modified k-w SST turbulence model to simulate wave run-up around a monopile subjected to regular waves using OpenFOAM", Coastal Engineering, 125, pp. 81-94, 2017. https://doi.org/10.1016/j.coastaleng.2017.04.004

[25] Larsen, B. E., Fuhrman, D. R. "On the over-production of turbulence beneath surface waves in Reynolds-averaged Navier-Stokes models", Journal of Fluid Mechanics, 853, pp. 419-460, 2018. https://doi.org/10.1017/jfm.2018.577

[26] Tu, J., Yeoh, G.-H., Liu, C. "Chapter 6 - Practical Guidelines for CFD Simulation and Analysis", In: Computational Fluid Dynamics, 2nd ed., Butterworth-Heinemann, Oxford, UK, 2013, pp. 219-273.

https://doi.org/10.1016/B978-0-08-098243-4.00006-8

[27] OpenFOAM Foundation "OpenFOAM - The Open Source CFD Toolbox - User Guide", OpenFOAM Ltd., Bracknell, UK, 2019.

[28] Gopala, V. R., van Wachem, B. G. M. "Volume of fluid methods for immiscible-fluid and free-surface flows", Chemical Engineering Journal, 141(1-3), pp. 204-221, 2008. https://doi.org/10.1016/j.cej.2007.12.035

[29] Deshpande, S. S., Anumolu, L., Trujillo, M. F. "Evaluating the performance of the two-phase flow solver interFoam", Computational Science \& Discovery, 5(1), Article number: 014016, 2012. https://doi.org/10.1088/1749-4699/5/1/014016

[30] Prasad, B., Hino, T., Suzuki K. "Numerical simulation of free surface flows around shallowly submerged hydrofoil by OpenFOAM", Ocean Engineering, 102, pp. 87-94, 2015. https://doi.org/10.1016/j.oceaneng.2015.04.049

[31] Askarizadeh, H., Ahmadikia, H., Ehrenpreis, C., Kneer, R., Pishevar, A., Rohlfs, W. "Heat transfer in the hydraulic jump region of circular free-surface liquid jets", International Journal of Heat and Mass Transfer, 146, Article number: 118823, 2020. https://doi.org/10.1016/j.ijheatmasstransfer.2019.118823
[32] Roenby, J., Bredmose, H., Jasak, H. "A computational method for sharp interface advection", Royal Society Open Science, 3(11), Article number: 160405, 2016. https://doi.org/10.1098/rsos.160405

[33] Laurila, E., Roenby, J., Maakala, V., Peltonen, P., Kahila, H., Vuorinen, V. "Analysis of viscous fluid flow in a pressure-swirl atomizer using large-eddy simulation", International Journal of Multiphase Flow, 113, pp. 371-388, 2019. https://doi.org/10.1016/j.ijmultiphaseflow.2018.10.008

[34] Larsen, B. E., Fuhrman, D. R., Roenby, J. "Performance of interFoam on the simulation of progressive waves", Coastal Engineering Journal, 61(3), pp. 380-400, 2019. https://doi.org/10.1080/21664250.2019.1609713

[35] Boussinesq, J. "Essai sur la théorie des eaux courantes (Essay on running water theory)", Mémoires présentés par divers savants à l'Académie des Sciences, Imprimerie Nationale, Paris, France, 1877. (in French)

[36] Wilcox, D. C. "Formulation of the $k-\omega$ Turbulence Model Revisited", AIAA Journal, 46(11), pp. 2823-2838, 2008. https://doi.org/10.2514/1.36541

[37] Menter, F. R. "Two-equation eddy-viscosity turbulence models for engineering applications", AIAA Journal, 32(8), pp. 1598-1605, 1994.

https://doi.org/10.2514/3.12149

[38] Eymard, R., Gallouët, T., Herbin, R. "Finite volume methods", Handbook of Numerical Analysis, 7, pp. 713-1020, 2000. https://doi.org/10.1016/S1570-8659(00)07005-8

[39] The Core Writing Team, Pachauri, R. K., Meyer, L. "Climate Change 2014, Synthesis Report", [pdf] Intergovernmental Panel on Climate Change, Geneva, Switzerland, 2014. Available at: https:/www.ipcc. ch/site/assets/uploads/2018/02/SYR_AR5_FINAL_full.pdf

[40] Wassmann, R., Hien, N. X., Hoanh, C. T., Tuong, T. P. "Sea Level Rise Affecting the Vietnamese Mekong Delta: Water Elevation in the Flood Season and Implications for Rice Production", Climatic Change, 66, pp. 89-107, 2004. https:/doi.org/10.1023/B:CLIM.0000043144.69736.b7

[41] Blum, M. D., Roberts, H. H. "Drowning of the Mississippi Delta due to insufficient sediment supply and global sea-level rise", Nature Geoscience, 2, pp. 488-491, 2009. https://doi.org/10.1038/ngeo553

[42] Maza, M., Lara, J. L., Losada, I. J. "Tsunami wave interaction with mangrove forests: A 3-D numerical approach", Coastal Engineering, 98, pp. 33-54, 2015. https://doi.org/10.1016/j.coastaleng.2015.01.002

[43] Truong, S. H., Ye, Q., Stive, M. J. F. "Estuarine Mangrove Squeeze in the Mekong Delta, Vietnam", Journal of Coastal Research, 33(4), pp. 747-763, 2017. https://doi.org/10.2112/JCOASTRES-D-16-00087.1

[44] Islam, M. R., Zhu, D. Z. "A Kernel Density-Based Algorithm to Despike ADV Data", Journal of Hydraulic Engineering, ASCE, 139(7), pp. 785-793, 2013. https://doi.org/10.1061/(ASCE)HY.1943-7900.0000734

[45] Botev, Z. I., Grotowski, J. F., Kroese, D. P. "Kernel Density Estimation via Diffusion", Annals of Statistics, 38(5), pp. 29162957, 2010.

https://doi.org/10.1214/10-AOS799 